\title{
Role of miR-223/paired box 6 signaling in temozolomide chemoresistance in glioblastoma multiforme cells
}

\author{
QUAN CHENG $^{1}$, XIAOQIANG MA ${ }^{2}$, HUI CAO $^{3}$, ZIGUI CHEN $^{1}$, XIN WAN $^{1}$, \\ RUI CHEN ${ }^{1}$, RENJUN PENG ${ }^{1}$, JUN HUANG ${ }^{1}$ and BING JIANG ${ }^{1}$ \\ ${ }^{1}$ Department of Neurosurgery, Xiangya Hospital, Central South University, Changsha, Hunan 410078; \\ ${ }^{2}$ Department of Neurosurgery, Traditional Chinese Medical Hospital of Xinjiang Uygur Autonomous Region, \\ Urumqi, Xinjiang 830000; ${ }^{3}$ Department of Neurosurgery, The Affiliated Hospital of \\ Hunan Traditional Chinese Medicine University, Changsha, Hunan 410000, P.R. China
}

Received November 4, 2015; Accepted November 4, 2016

DOI: $10.3892 / \mathrm{mmr} .2016 .6078$

\begin{abstract}
Glioblastoma (GBM) is the predominant and most fatal type of brain tumor in adults. The prognosis of GBM remains poor despite advances in surgery, chemotherapy and radiotherapy. It is common that patients with GBM exhibit innate or acquired resistance to temozolomide (TMZ), a standard chemotherapeutic agent for GBM, and a previous report demonstrated that miRNA-233 (miR-223) promotes the growth and invasion of GBM cells by targeting tumor suppressor paired box 6 (PAX6). The present study explored the effect of TMZ on miR-223/PAX6 signaling in addition to the effect of miR-223/PAX6 signaling on TMZ chemoresistance in human GBM cells. Luciferase reporter assays confirmed that miR-223 directly targets PAX6 through binding to its 3'-untranslated region. TMZ reduced the expression level of $\mathrm{miR}-223$ in a concentration-dependent manner in U251 and U118 GBM cells, which led to increased expression of PAX6. miR-223 and/or PAX6 were overexpressed and knocked down in U251 and U118 cells, and the half maximal inhibitory concentration $\left(\mathrm{IC}_{50}\right)$ of TMZ and cell proliferation under TMZ treatment were used as measures of TMZ chemoresistance. The results demonstrated that overexpression of miR-223 in GBM cells markedly decreased TMZ-induced inhibition of cell proliferation and increased $\mathrm{TMZ} \mathrm{IC}_{50}$, which could be abolished by overexpression of PAX6. On the other hand, knocking down miR-223 in GBM cells with antagomir significantly enhanced the inhibitory effect of TMZ on GBM cell proliferation and decreased the $\mathrm{TMZ} \mathrm{IC}_{50}$, which could be abolished by knockdown of PAX6. In conclusion, the present study demonstrated
\end{abstract}

Correspondence to: Dr Bing Jiang or Dr Jun Huang, Department of Neurosurgery, Xiangya Hospital, Central South University, 87 Xiangya Road, Changsha, Hunan 410078, P.R. China

E-mail: csubingjiang@163.com

E-mail: 13508488502@163.com

Key words: miR-223, paired box 6, glioblastoma multiforme, temozolomide, chemoresistance, proliferation that TMZ inhibits GBM cell proliferation by inhibiting the expression of miR-223, which leads to increased expression of tumor suppressor PAX6. Overexpression of miR-223 increases TMZ chemoresistance, while inhibition of miR-223 with antagomir markedly decreases TMZ chemoresistance in GBM cells. The present study provided novel insight into the molecular mechanisms underlying the pharmacological effects, in addition to the chemoresistance, of TMZ for GBM.

\section{Introduction}

Glioblastoma multiforme (GBM) is the most malignant type of primary adult brain tumor (1). Patients with GBMs have a median survival of only 12-15 months (2). Surgery with maximum feasible resection, radiotherapy and treatment with alkylating agent temozolomide (TMZ) are standard treatments for GBM (3). The rapid growth and high rate of recurrence of GBM make surgical resection non-curative and leads to poor prognosis of this disease (4). Although TMZ has been identified to improve the survival rates of patients with GBM, a majority experience disease progression within one year (3). Therefore, it is important to identify ways to promote the efficacy of TMZ for GBM.

Paired box 6 (PAX6), a transcription factor critical for the development of the brain, is additionally expressed in the adult brain (5). Previous studies have demonstrated that PAX6 functions as a tumor suppressor that markedly limits the growth of GBM cells (6). The expression level of PAX6 reportedly is inversely correlated with the tumorigenicity and invasion of GBM cell lines and is significantly reduced in GBMs $(7,8)$.

MicroRNAs (miRNAs) are small non-coding RNA molecules (containing 22 nucleotides) that function in RNA silencing and post-transcriptional regulation of gene expression. miRNAs normally cleave mRNA by base-pairing to the 3'-untranslated region (UTR) of the target genes (9). Previously, it has been identified that miRNAs are involved in the progression and chemoresistance of numerous types of cancer, including GBM $(10,11)$. Previous studies have suggested that miRNAs may be important regulators of the therapeutic efficacy of TMZ for GBMs (12-14). 
The present study aimed to investigate the potential interaction among TMZ, PAX6 and miRNAs in GBM cells and assess its impact on GBM cell proliferation for, to the best of our knowledge, the first time.

\section{Materials and methods}

Cells lines and reagents. U251 and U118 human GBM cell lines were purchased from the Cell Center of Peking Union Medical University (Beijing, China) and American Type Culture Collection (ATCC; Manassas, VA, USA), respectively. Human PAX6-short hairpin RNA (shRNA) plasmid (sc-36195-SH), control shRNA plasmid (sc-108060), mouse monoclonal anti-PAX6 (AD2, 35) (sc-53108) and mouse monoclonal anti- $\beta$-actin (C4) (sc-47778) antibodies were purchased from Santa Cruz Biotechnology, Inc. (Santa Cruz, CA, USA). miR-223 mimic and miR-223 antagomir [a 20-O-methyl (20-OMe) modified antisense oligonucleotide 5'-GGGGUAUUUGACAAACUGACA-3'] were purchased from Shanghai GenePharma Co., Ltd. (Shanghai, China). Lipofectamine 2000 transfection reagent was purchased from Life Technologies (Thermo Fisher Scientific, Inc., Waltham, MA, USA). The bromodeoxyuridine (BrdU) Cell Proliferation ELISA kit (colorimetric; ab126556) was purchased from Abcam (Cambridge, MA, USA) and the dual-luciferase reporter assay system was purchased from Promega Corp. (Madison, WI, USA). Human full length PAX6 cDNA clone (SC109551) was purchased from OriGene Technologies, Inc. (Beijing, China) and subcloned into pcDNA 3.1 expression vector (Life Technologies; Thermo Fisher Scientific, Inc.). The PAX6-3'UTR-luciferase reporter was generated by subcloning human PAX6 3'-UTR into the psiCheck 2 vector (Promega Corp.) downstream of the Renilla luciferase gene. miRNAs potentially able to suppress PAX6 expression were selected using TargetScan prediction software version 6.0 (www.targetscan.org). TMZ and all chemicals of reagent grade were purchased from Sigma-Aldrich (Merck Millipore, Darmstadt, Germany). TMZ was dissolved in dimethyl sulfoxide at a stock concentration of $100 \mathrm{mM}$ and stored at $-20^{\circ} \mathrm{C}$.

Transfection. Plasmids, miR-223 mimic and antagomir were respectively transfected into cells using Lipofectamine 2000 transfection reagent (Life Technologies; Thermo Fisher Scientific, Inc.) according to the manufacturer's instructions. The cells were subject to analysis $48 \mathrm{~h}$ after transfection.

Western blot analysis. Cells were lysed with a hypotonic buffer containing $2 \%$ Nonidet-P and a protease inhibitor cocktail (Sigma-Aldrich; Merck Millipore) by sonication three times for $3 \mathrm{sec}$ on ice. The supernatant obtained after centrifugation at $2,000 \mathrm{x} \mathrm{g}$ for $15 \mathrm{~min}$ at $4^{\circ} \mathrm{C}$ was used for protein concentration determination by the Coomassie blue method and for subsequent steps. Equal amounts of protein $(5 \mu \mathrm{g})$ for each sample were separated using a 10\% SDS-polyacrylamide gel and blotted onto a polyvinylidene difluoride microporous membrane (EMD Millipore, Billerica, MA, USA). Membranes were incubated for $1 \mathrm{~h}$ at room temperature with a 1:1,000 dilution of the primary antibody and then washed and revealed using incubation with bovine anti-mouse secondary antibody conjugated with horseradish peroxidase conjugate (1:5,000; Santa Cruz Biotechnology, Inc.; cat. no. sc-2371) at room temperature for $1 \mathrm{~h}$. Peroxidase was observed using a GE Healthcare ECL kit (RPN2235; GE Healthcare Life Sciences, Shanghai, China). Three independent experiments were performed.

Reverse transcription-quantitative polymerase chain reaction $(R T-q P C R)$. RNA was prepared from cells using TRIzol reagent and cDNAs were synthesized using SuperScript II reverse transcriptase (Invitrogen; Thermo Fisher Scientific, Inc.). RT-qPCR was performed on an ABI-Prism 7700 Sequence Detection system, with use of the fluorescent dye SYBR-Green Master Mix (Applied Biosystems, Thermo Fisher Scientific, Inc., Beijing, China) as described by the manufacturer. The results were normalized against that of the reference gene glyceraldehyde-3-phosphate dehydrogenase (GAPDH) in the same sample. The primers used were as follows: Human PAX6, 5'-AGACACAGCCCTCACAAAC-3' (forward) and 5'-ATCATAACTCCGCCCATTC-3' (reverse); human GAPDH, 5'-GACTCATGACCACAGTCCATGC-3' (forward) and 5'-AGAGGCAGGGATGATGTTCTG-3' (reverse). The PCR reaction mixture contained $12.5 \mu \mathrm{l}$ SYBR-Green Master Mix (Thermo Fisher Scientific, Inc.), $500 \mathrm{ng}$ template cDNA, forward and reverse primers $(0.25 \mu \mathrm{M}$ each) and $12 \mu 1$ nuclease-free water (Thermo Fisher Scientific, Inc.). The thermocycling conditions were as follows: $20 \mathrm{sec}$ at $95^{\circ} \mathrm{C}$; followed by 40 cycles of $5 \mathrm{sec}$ at $95^{\circ} \mathrm{C}$ and $30 \mathrm{sec}$ at $60^{\circ} \mathrm{C}$. Each experiment was repeated three times in duplicate.

Luciferase assay. Cells were transfected with the human PAX6-3'UTR-luciferase reporter plasmid using Lipofectamine 2000 transfection reagent (Life Technologies; Thermo Fisher Scientific, Inc.) and then cultured for $48 \mathrm{~h}$. Luciferase assays were performed with the Dual-Luciferase Reporter Assay system (Promega Corp.) according to the manufacturer's instructions. Each experiment was repeated three times in duplicate.

BrdU cell proliferation assay. Cells were cultured at $2 \times 10^{5}$ cells per well in 96-well tissue culture plates and treated with TMZ $(400 \mu \mathrm{mol} / \mathrm{l})$ for $48 \mathrm{~h}$ at $37^{\circ} \mathrm{C}$. Cell proliferation was measured at $48 \mathrm{~h}$ with a colorimetric BrdU Cell Proliferation ELISA kit (Abcam) $(15,16)$. BrdU was added $4 \mathrm{~h}$ before the end of the incubation period. The cells were then fixed, the DNA was denatured, and BrdU content was assessed using a monoclonal anti-BrdU antibody following the manufacturer's instructions (Abcam). Cell proliferation was presented as the optical density values at $450 \mathrm{~nm}$. Each experiment was repeated three times in duplicate.

TMZ chemosensitivity assay. Cells were plated in duplicate in 96-well plates at a density of 5,000 cells per well. Transfection of plasmids, miR-223 mimic and antagomir were performed $6 \mathrm{~h}$ later. After $24 \mathrm{~h}$ of incubation, the medium was replaced by fresh medium with or without various concentrations ( 0.1 , $0.15,0.20,0.25,0.3,0.5,1.0,1.5,3.0,6.0$ or $15.0 \mathrm{mM}$ ) of TMZ (Sigma-Aldrich; Merck Millipore). Then cell viability was assayed $72 \mathrm{~h}$ later using a modified MTT assay as previously described (17). The half maximal inhibitory concentration 
A

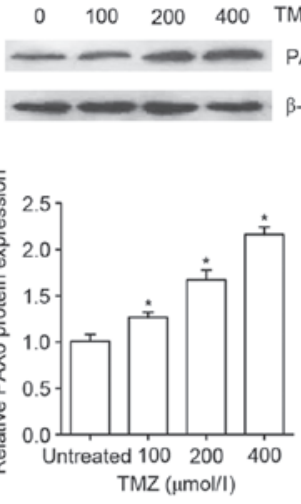

B

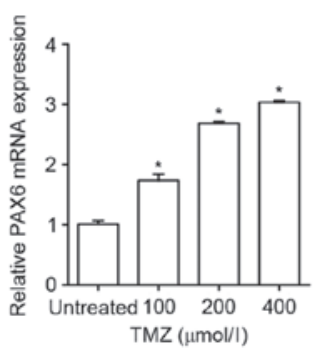

$0 \quad 100 \quad 200 \quad 400 \quad \mathrm{TMZ}(\mu \mathrm{mol} / \mathrm{l})$
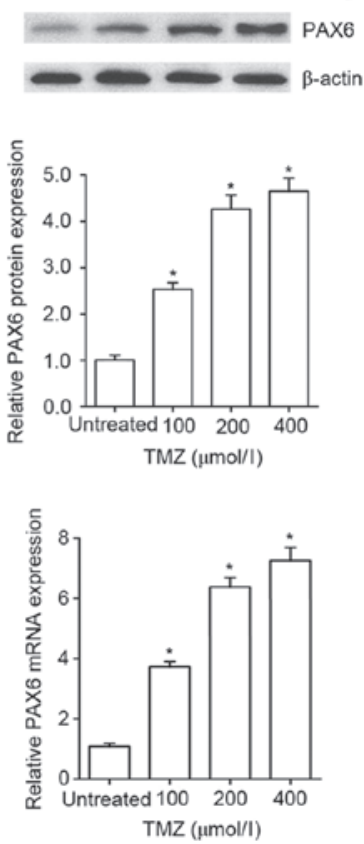

Figure 1. Effect of TMZ on expression of PAX6 in GBM cells. The (A) protein and (B) mRNA levels of PAX6 were determined with western blot analysis and reverse transcription-quantitative polymerase chain reaction in U251 (left panel) and U118 (right panel) GBM cells treated with the indicated concentrations of TMZ for $48 \mathrm{~h}$. $\beta$-actin blotting was used as a loading control in western blot analysis. Density of the PAX6 blot was normalized against that of the $\beta$-actin blot to obtain a relative blot density, which was then expressed as fold changes to that of the untreated cells (designated as 1). The mRNA level of PAX6 was also expressed as fold changes to that of the untreated cells (designated as 1). ${ }^{*} \mathrm{P}<0.05$ vs. untreated. TMZ, temozolomide; PAX6, paired box 6; GBM, glioblastoma multiforme.

$\left(\mathrm{IC}_{50}\right)$ values were defined as the concentrations resulting in a $50 \%$ reduction in growth compared with control cell growth.

Statistical analysis. Statistical analyses were performed with SPSS for Windows, version 10.0 (SPSS Inc., Chicago, IL, USA). All data values were expressed as the mean \pm standard deviation. Comparisons of the means among multiple groups were performed with one-way analysis of variance followed by post hoc pairwise comparisons using Tukey's tests. A two-tailed $\mathrm{P}<0.05$ was considered to indicate a statistically significant difference.

\section{Results}

TMZ induces expression of PAX6 in GBM cells. To examine the effect of TMZ on the expression of PAX6 in GBM cells, human U251 and U118 GBM cells were treated with TMZ in increasing concentrations $(100,200$ or $400 \mu \mathrm{M})$ for $48 \mathrm{~h}$. As presented in Fig. 1, TMZ in the concentration range of $100-400 \mu \mathrm{M}$ increasingly augmented the protein and mRNA levels of PAX6 in U251 and U118 cells within 48 h of treatment. The results indicate that TMZ induces the expression of PAX6 in GBM cells.

PAX6 is critical for the inhibitory effect of TMZ on GBM cell proliferation. To explore the functional role of PAX6 in the inhibitory effect of TMZ on GBM cell proliferation, PAX6 was

A
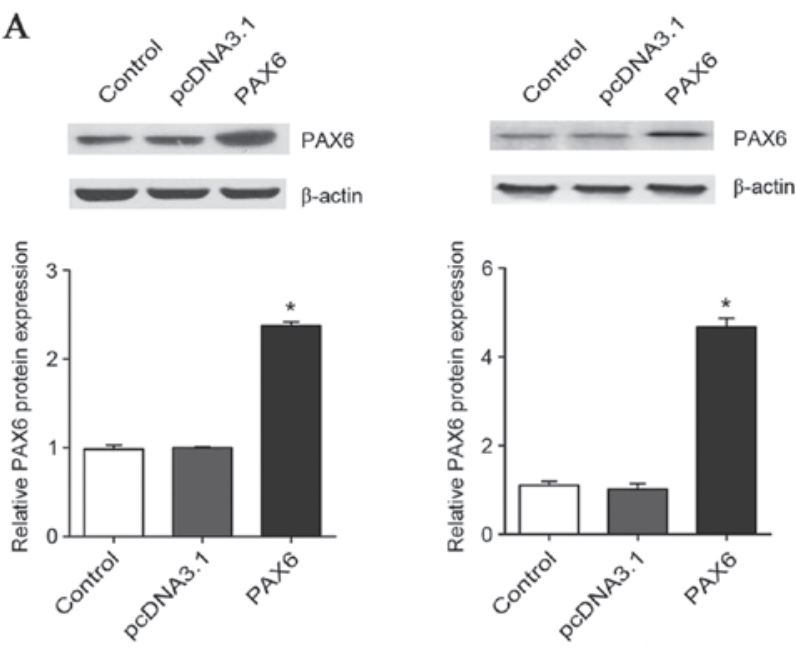

B
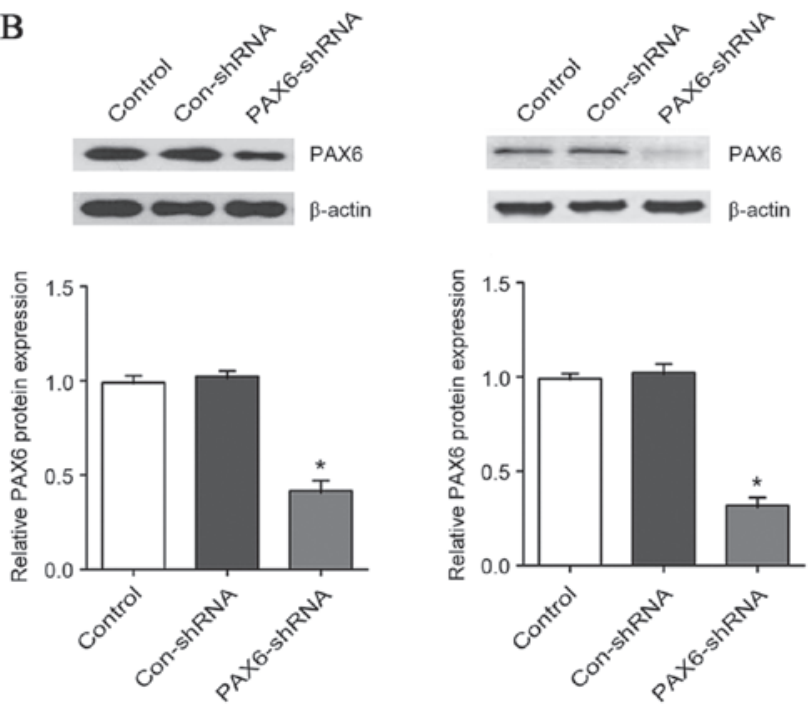

Figure 2. Overexpression and knockdown of PAX6 in GBM cells. (A) In U251 (left panel) and U118 (right panel) GBM cells, the protein levels of PAX6 were determined by western blot analysis in control cells, cells transfected with the empty pcDNA3.1 plasmid and cells transfected with the PAX6 expression vector. (B) In U251 (left panel) and U118 (right panel) GBM cells, the protein levels of PAX6 were determined with western blot analysis in control cells, cells transfected with Con-shRNA and cells transfected with PAX6-shRNA plasmids. $\beta$-actin blotting was used as a loading control. Density of the PAX6 blot was normalized against that of the $\beta$-actin blot to obtain a relative blot density, which was then expressed as fold changes to that of control cells (designated as 1). "P<0.05 vs. control. PAX6, paired box 6; GBM, glioblastoma multiforme; Con, control; shRNA, short hairpin RNA.

overexpressed and knocked down in U251 and U118 cells. As presented in Fig. 2, transfection of the cells with a PAX6 expression vector increased the protein level of PAX6 by 2.4 -fold in U251 cells and 4.7-fold in U118 cells. On the other hand, transfection of the cells with PAX6-shRNA plasmids knocked down the protein level of PAX6 by $\sim 60 \%$ in U251 cells and $70 \%$ in U118 cells. The proliferation of U251 and U118 cells with or without TMZ $(400 \mu \mathrm{M})$ treatment was measured after this. BrdU cell proliferation assays demonstrated that $48 \mathrm{~h}$ of TMZ treatment significantly inhibited GBM cell proliferation in both U251 and U118 cells (Fig. 3). Overexpression of PAX6 increased the inhibitory effect of TMZ on GBM cell proliferation; on the other hand, knockdown of PAX6 abolished the inhibitory effect of TMZ on GBM cell proliferation (Fig. 3). 

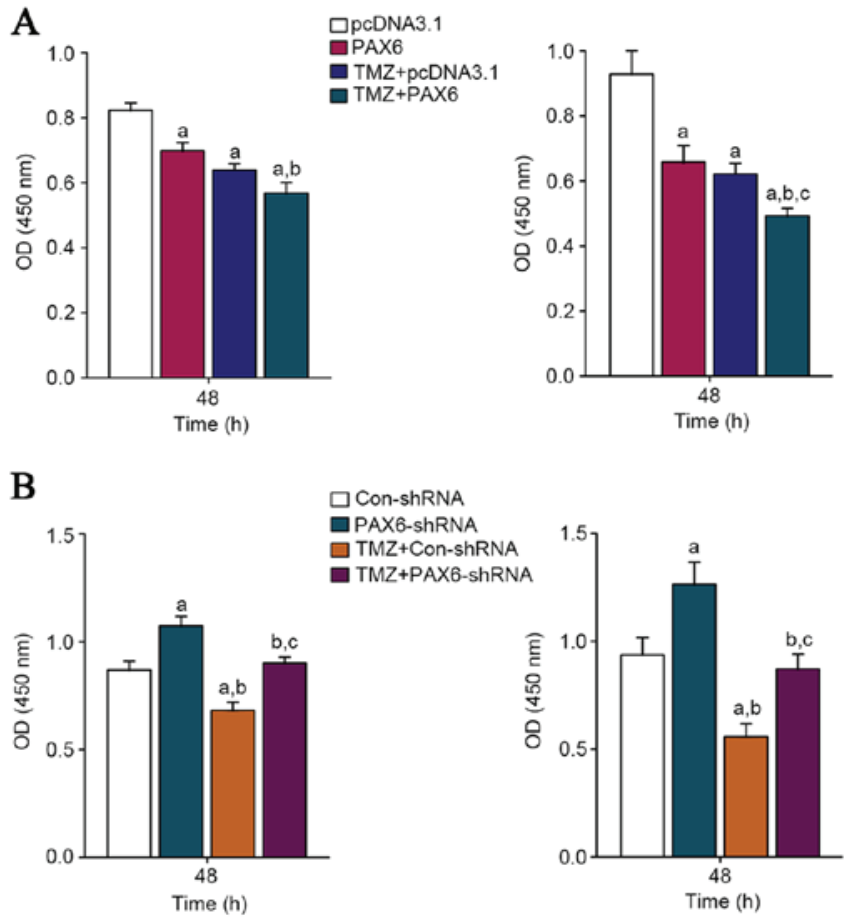

Figure 3. Effect of PAX6 on proliferation of GBM cells. (A) In U251 (left panel) and U118 (right panel) GBM cells, cell proliferation was measured with a microplate reader-based BrdU cell proliferation ELISA kit in cells transfected with the empty pcDNA3.1 plasmid and cells transfected with the PAX6 expression vector with or without TMZ treatment $(400 \mu \mathrm{M})$ for $48 \mathrm{~h}$. Cell proliferation was shown in OD values at $450 \mathrm{~nm}$. ${ }^{\mathrm{a}} \mathrm{P}<0.05 \mathrm{vs}$. pcDNA3.1; ${ }^{\mathrm{b}} \mathrm{P}<0.05$ vs. PAX6; ${ }^{\mathrm{P}} \mathrm{P}<0.05$ vs. TMZ + pcDNA3.1. (B) In U251 (left panel) and U118 (right panel) GBM cells, cell proliferation was measured with a microplate reader-based BrdU cell proliferation ELISA kit in cells transfected with Con-shRNA and cells transfected with PAX6-shRNA plasmids with or without TMZ treatment $(400 \mu \mathrm{M})$ for $48 \mathrm{~h}$. Cell proliferation was presented as the $\mathrm{OD}$ values at $450 \mathrm{~nm}$. ${ }^{\mathrm{a}} \mathrm{P}<0.05$ vs. Con-shRNA; ${ }^{\mathrm{b}} \mathrm{P}<0.05$ vs. PAX6-shRNA; ${ }^{\mathrm{P}}<0.05$ vs. TMZ + Con-shRNA. PAX6, paired box 6 ; GBM, glioblastoma multiforme; TMZ, temozolomide; OD, optical density; Con, control; shRNA, short hairpin RNA.

The results suggest that PAX6 is an essential mediator of the inhibitory effect of TMZ on GBM cell proliferation.

TMZ inhibits expression of miR-223, which targets PAX6 in GBM cells. Next the TargetScan prediction software was employed to analyze the 3'-UTR of the PAX6 gene, which revealed that miR-223, miR-590-3p, miR-190, miR-190b and miR-7 would be most likely to target PAX6. As presented in Fig. 4A, TMZ (100-400 $\mu \mathrm{M})$ concentration-dependently reduced the expression of miR-223 in U251 and U118 cells in a concentration-dependent manner within $48 \mathrm{~h}$ of treatment. Compared with that of miR-223, the expression levels of miR-590-3p, miR-190, miR-190b and miR-7 were not as significantly regulated by TMZ in GBM cells (data not shown). Thus, the interaction among TMZ, PAX6 and miR-223 in GBM cells was further explored. To demonstrate a direct interaction between miR-223 and PAX6, the 3'-UTR of the PAX6 gene was inserted downstream of the Renilla luciferase gene in the psiCheck 2 vector to generate a wild type (wt)-PAX6-3'UTR-luciferase reporter. Meanwhile, the potential binding sequence for miR-223 in the 3'-UTR of the PAX6 gene, as predicted by TargetScan, was mutated to generate a mut-PAX6-3'UTR-luciferase reporter (Fig. 4B). U251
A
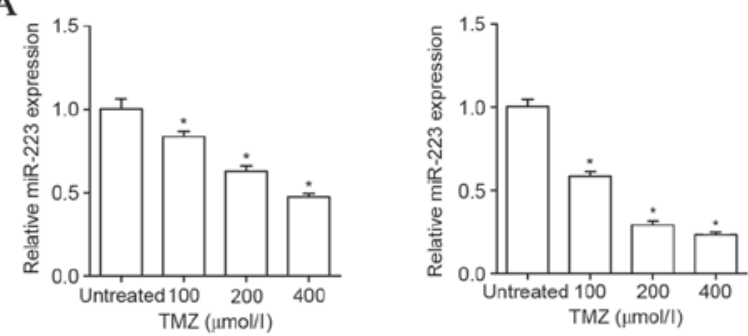

B

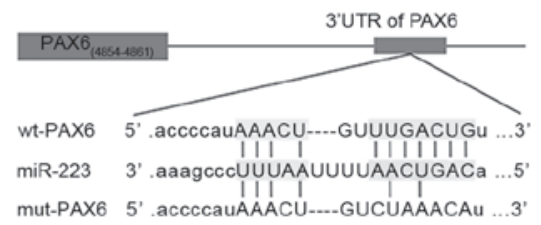

$\mathrm{C}$
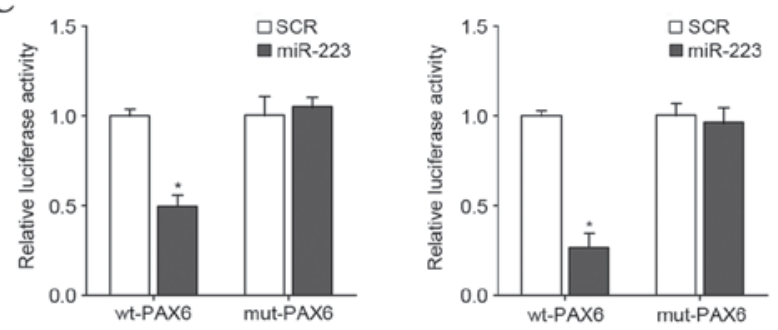

D
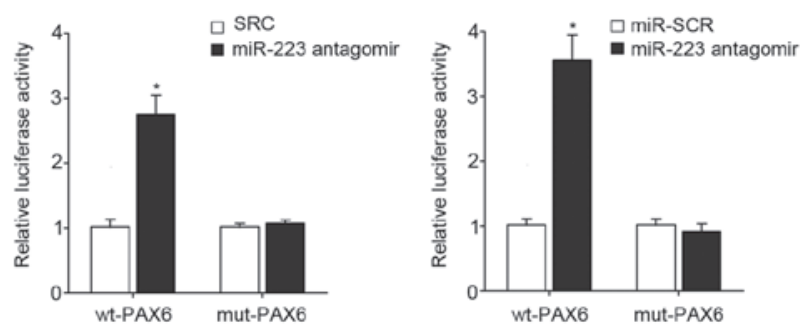

Figure 4. Effect of TMZ on expression of miR-223 and effect of miR-223 on PAX6 3'-UTR luciferase reporter in GBM cells. (A) miR-223 levels were determined in U251 (left panel) and U118 (right panel) GBM cells treated with the indicated concentrations of TMZ for $48 \mathrm{~h}$. ${ }^{*} \mathrm{P}<0.05$ vs. untreated. (B) miR-223 binding sequence in wt-PAX6-3'UTR-luciferase reporter and mutated sequence in mut-PAX6-3'UTR-luciferase reporter. (C) Luciferase activities were measured in U251 (left panel) and U118 (right panel) GBM cells co-transfected with miR-223 mimic and the PAX6 3'-UTR luciferase reporter with wt-PAX6 or mut-PAX6 miR223-binding sequences. Cells co-transfected with SCR instead of miR-223 mimic were used as the control. The luciferase activity was expressed as fold changes to that of cells co-transfected with SCR (designated as 1). (D) Luciferase activities were measured in U251 (left panel) and U118 (right panel) GBM cells co-transfected with miR-223 antagomir and PAX6 3'-UTR luciferase reporter with wt-PAX6 or mut-PAX6 3'-UTR sequence. Cells co-transfected with SCR instead of miR-223 antagomir were used as the control. The luciferase activity was expressed as fold changes to that of cells co-transfected with SCR (designated as 1). "P<0.05 vs. SCR. TMZ, temozolomide; PAX6, paired box 6; UTR, untranslated region; GBM, glioblastoma multiforme; miR, miRNA; wt, wild type; mut, mutated; SCR, scrambled control.

and U118 cells were co-transfected with miR-223 mimic or antagomir together with either the wt-PAX6-3'UTR-luciferase reporter or the mut-PAX6-luciferase reporter. As presented in Fig. 4C and D, compared with the scrambled control, miR-223 mimic and antagomir decreased and increased the luciferase activity of the wt-PAX6-3'UTR respectively, however did not affect that of the mut-PAX6-3'UTR reporter. This suggests that miR-223 could suppress the expression of PAX6 by directly 
A

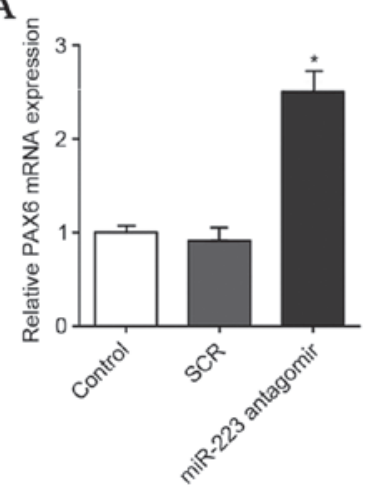

B

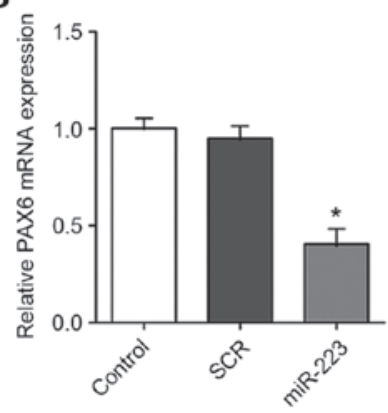

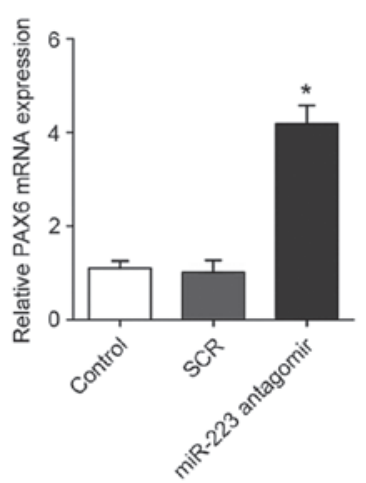

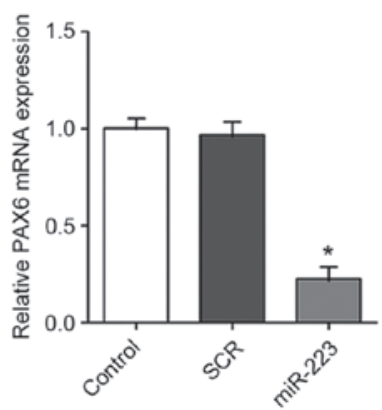

Figure 5. Effect of miR-223 on the mRNA level of PAX6 in GBM cells (A) In U251 (left panel) and U118 (right panel) GBM cells, the mRNA levels of PAX6 were determined with RT-qPCR in control cells, cells transfected with scrambled control (SCR), and cells transfected with miR-223 antagomir. (B) In U251 (left panel) and U118 (right panel) GBM cells, the mRNA levels of PAX6 were determined by RT-qPCR in control cells, cells transfected with SCR and cells transfected with miR-223 mimic. The mRNA level of PAX6 was expressed as fold changes to that of control (designated as 1 ). ${ }^{*} \mathrm{P}<0.05$ vs. control. PAX6, paired box 6; GBM, glioblastoma multiforme; RT-qPCR, reverse transcription-quantitative polymerase chain reaction; SCR, scrambled control; miR, miRNA.

binding to the 3'-UTR of the PAX6 gene. RT-qPCR assays demonstrated that miR-223 mimic and antagomir decreased and increased the mRNA levels of PAX6, respectively, in U251 and U118 cells (Fig. 5), confirming that miR-223 targets PAX6 in GBM cells.

TMZ/miR-223 signaling inhibits GBM cell proliferation through inducing expression of PAX6. The above observations indicate that TMZ inhibits the expression of miR-223, which targets PAX6 in GBM cells. To explore the effect of TMZ/miR-223/PAX6 signaling on GBM cell proliferation, it was first examined how TMZ/miR-223 signaling would regulate the expression of PAX6 in GBM cells. In the absence of TMZ treatment, compared with scrambled control, miR-223 mimic decreased the expression of PAX6 by approximately $60 \%$ in U251 cells and $70 \%$ in U118 cells, while miR-223 antagomir increased the expression of PAX by approximately 2.1-fold in U251 cells and 2.7-folds in U118 cells (Fig. 6). In the presence of TMZ treatment ( $400 \mu \mathrm{M}$ for $48 \mathrm{~h}$ ), compared with scrambled control, miR-223 mimic decreased the expression of PAX6 by approximately $45 \%$ in U251 cells and $75 \%$ in U118 cells, while miR-223 antagomir increased the expression of PAX by approximately 2.5-fold in U251 cells and 3.5-fold in U118 cells (Fig. 6). Regardless of TMZ treatment, overexpression and knockdown of PAX6 respectively abolished the effects

of miR-223 mimic and antagomir (Fig. 6). In the presence of TMZ treatment ( $400 \mu \mathrm{M}$ for $48 \mathrm{~h}$ ), compared with the controls, miR-223 mimic increased proliferation by approximately 1.3-fold in U251 cells and 1.6-fold in U118 cells, while miR-223 antagomir decreased proliferation by approximately $30 \%$ in U251 cells and 43\% in U118 cells (Fig. 7). Overexpression and knockdown of PAX6, respectively, abolished the effects of miR-223 mimic and antagomir (Fig. 7).

Inhibition of miR-223/PAX6 signaling increases TMZ chemoresistance in GBM cells. To explore the effect of inhibition of miR-223/PAX6 signaling on TMZ chemoresistance in GBM cells, TMZ $\mathrm{IC}_{50}$ values were examined. A higher $\mathrm{IC}_{50}$ value was considered to correspond with clinical chemoresistance to TMZ. U251 and U118 GBM cells were plated in 96-well plates. Transfection of plasmids, miR-223 mimic and antagomir were performed $6 \mathrm{~h}$ later. After $24 \mathrm{~h}$ of incubation, the medium was replaced by fresh medium with or without various concentrations of TMZ, and cell viability was assayed $72 \mathrm{~h}$ later. As presented in Fig. 8, the TMZ $\mathrm{IC}_{50}$ values in U251 and U118 cells were 240 and $970 \mu \mathrm{M}$, respectively; miR-223 mimics increased the $\mathrm{TMZ} \mathrm{IC}_{50}$ to 2,549 and $8,900 \mu \mathrm{M}$, respectively, which was abolished by overexpression of PAX6. On the other hand, inhibiting miR-223 with antagomir decreased TMZ $\mathrm{IC}_{50}$ to 45 and $170 \mu \mathrm{M}$ in $\mathrm{U} 251$ and U118 cells, respectively, which was abolished by knockdown of PAX6 (Fig. 8).

\section{Discussion}

GBM is a fatal adult brain tumor (18), and the prognosis of GBM remains poor despite advances in surgery, chemotherapy and radiotherapy $(2,4)$. It is common that patients with GBM show innate or acquired resistance to TMZ, a standard chemotherapeutic agent for GBM (3). PAX6 is reported as an independent prognostic marker for GBM (5). There is accumulating evidence that PAX6 functions as a tumor suppressor and suppresses growth of GBM cells (6). A recent study has demonstrated that miR-223 promotes the growth and invasion of GBM cells by targeting PAX6 (8). The present study confirms that miR-223 directly targets PAX6 in GBM cells. Nevertheless, the highlight of the present study is providing the first evidence that TMZ inhibits the proliferation of GBM cells by increasing the expression of PAX6 through inhibiting miR-223. In addition, it demonstrates that inhibiting miR-223 can markedly decrease TMZ chemoresistance in GBM cells, suggesting that miR-223/PAX6 signaling could be a potential target for overcoming TMZ chemoresistance in GBM.

In the present study, two GBM cell lines (U251 and U118) were used as the cell models. In both cell lines, TMZ concentration-dependently decreased the expression of miR-223, which led to increased expression of PAX6 and decreased proliferation of GBM cells. The TMZ/miR-223/PAX signaling axis provides novel insight into the pharmacological mechanisms of TMZ. The molecular mechanism underlying TMZ-induced inhibition of miR-223 requires further investigation.

TMZ $\mathrm{IC}_{50}$ was employed as a measure of TMZ chemoresistance in GBM cells. An increased $\mathrm{IC}_{50}$ was considered to correspond with clinical chemoresistance to TMZ. Overexpression and inhibition of miR-223, respectively, decreased and increased the expression of PAX6, which 
A

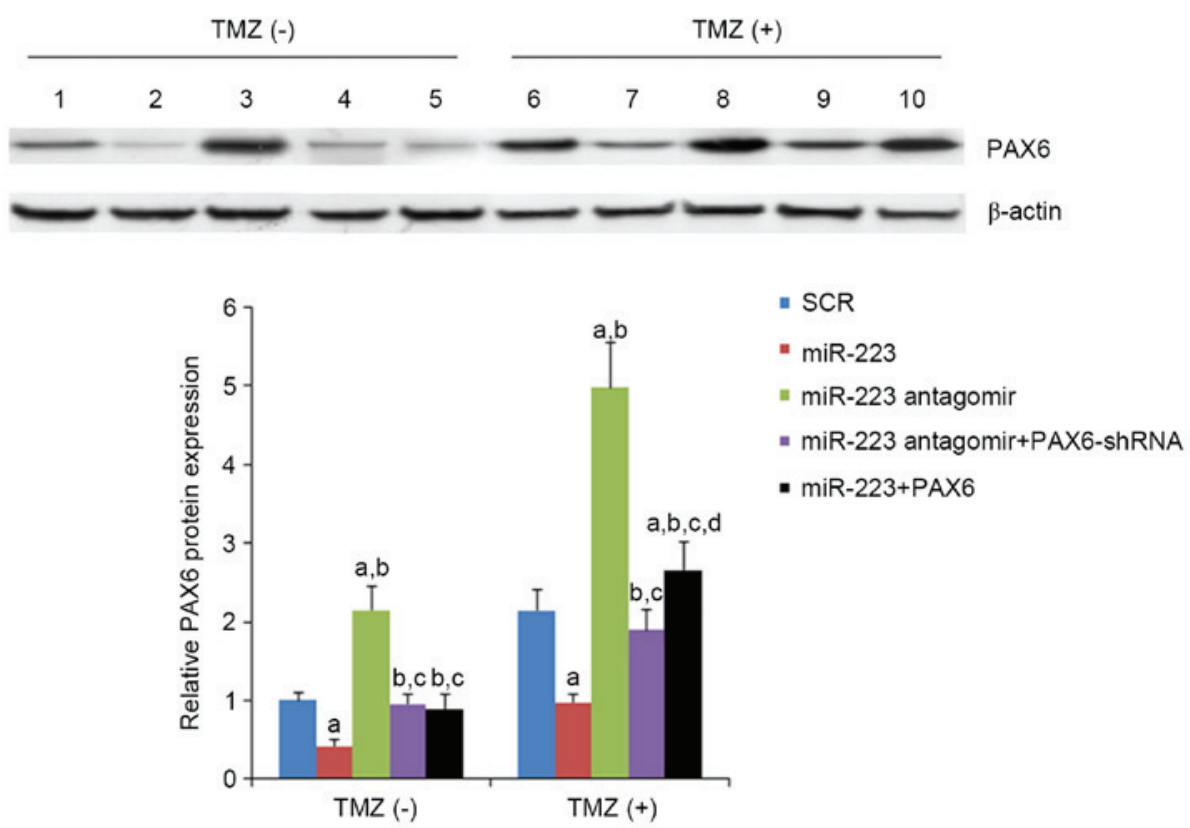

B
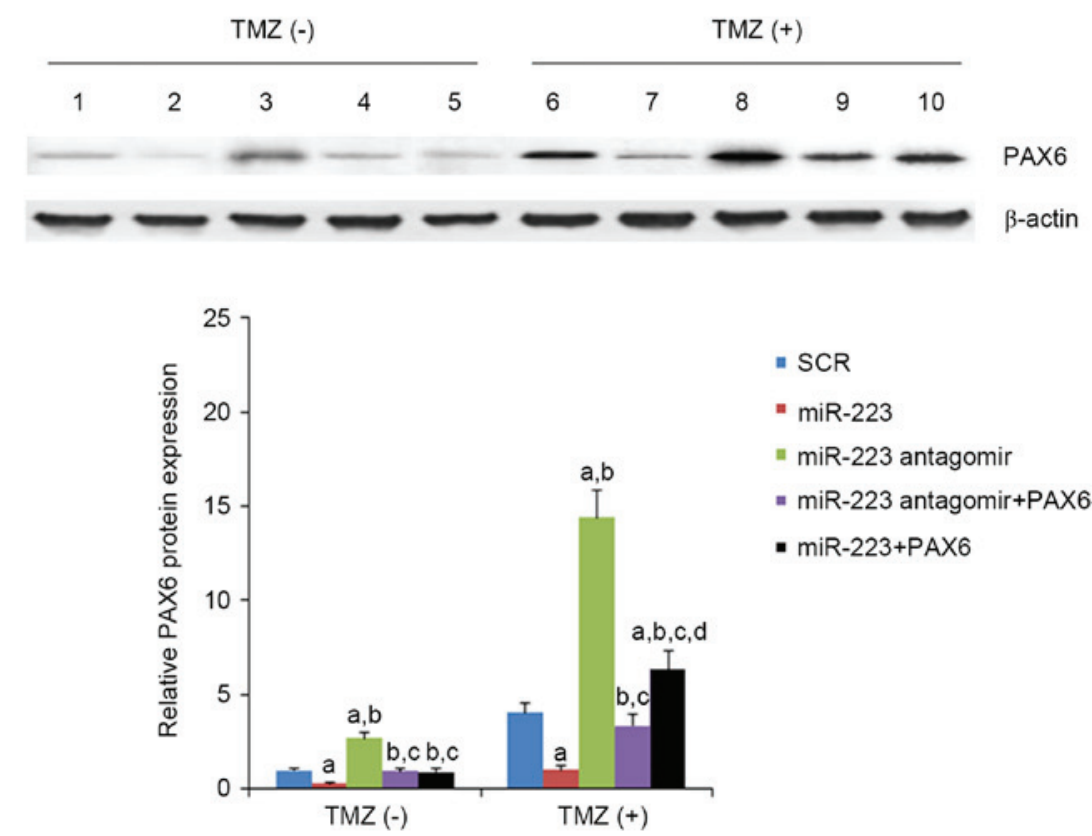

- SCR

- miR-223

" miR-223 antagomir

- miR-223 antagomir+PAX6-shRNA

- miR-223+PAX6

Figure 6. Effect of TMZ/miR-223 signaling on the protein level of PAX6 in GBM cells. In (A) U251 and (B) U118 GBM cells with or without TMZ treatment $(400 \mu \mathrm{mol} / 1)$ for $48 \mathrm{~h}$, the protein levels of PAX6 were determined with western blot analysis in cells transfected with SCR (lanes 1 and 6), cells transfected with miR-223 mimic (lanes 2 and 7), cells transfected with miR-223 antagomir (lanes 3 and 8), cells co-transfected with miR-223 antagomir and PAX6-shRNA (lanes 4 and 9) and cells co-transfected with miR-223 mimic and PAX6 (lanes 5 and 10). $\beta$-actin blotting was used as a loading control. Density of the PAX6 blot was normalized against that of the $\beta$-actin blot to obtain a relative blot density, which was then expressed as fold changes to that of cells transfected with SCR without TMZ treatment (designated as 1 ). ${ }^{a} \mathrm{P}<0.05$ vs. SCR; ${ }^{b} \mathrm{P}<0.05$ vs. miR-223; ${ }^{\mathrm{c}} \mathrm{P}<0.05$ vs. miR-223 antagomir; ${ }^{\mathrm{d}} \mathrm{P}<0.05$ vs. miR-223 antagomir + PAX6-shRNA. TMZ, temozolomide; miR, miRNA; PAX6, paired box 6; GBM, glioblastoma multiforme; SCR, scrambled control; shRNA, short hairpin RNA.

markedly altered $\mathrm{TMZ} \mathrm{IC}_{50}$ by $\sim 1$ order of magnitude in GBM cells. In the presence of TMZ, miR-223 antagomir significantly enhanced TMZ-induced inhibition on GBM cell proliferation and decreased TMZ chemoresistance, suggesting that inhibition of miR-223 may be a potential novel strategy to enhance the therapeutic effects of TMZ on GBM. The effects of miR-223 antagomir was respectively abolished and enhanced by knockdown and overexpression of PAX6, confirming that miR-223 promotes GBM resistance to TMZ predominantly by downregulating PAX6, or miR-223 antagomir decreases TMZ chemoresistance by upregulating PAX6.

miR-223 is a highly conserved miRNA, that was originally identified to be crucial for myeloid differentiation of progenitor cells (19). The expression levels of miR-223 are reportedly decreased in chronic lymphocytic leukemia (CLL) and could predict treatment-free survival and overall survival for CLL (20). It is also commonly repressed in hepatocellular carcinoma (21). A previous study has demonstrated that 

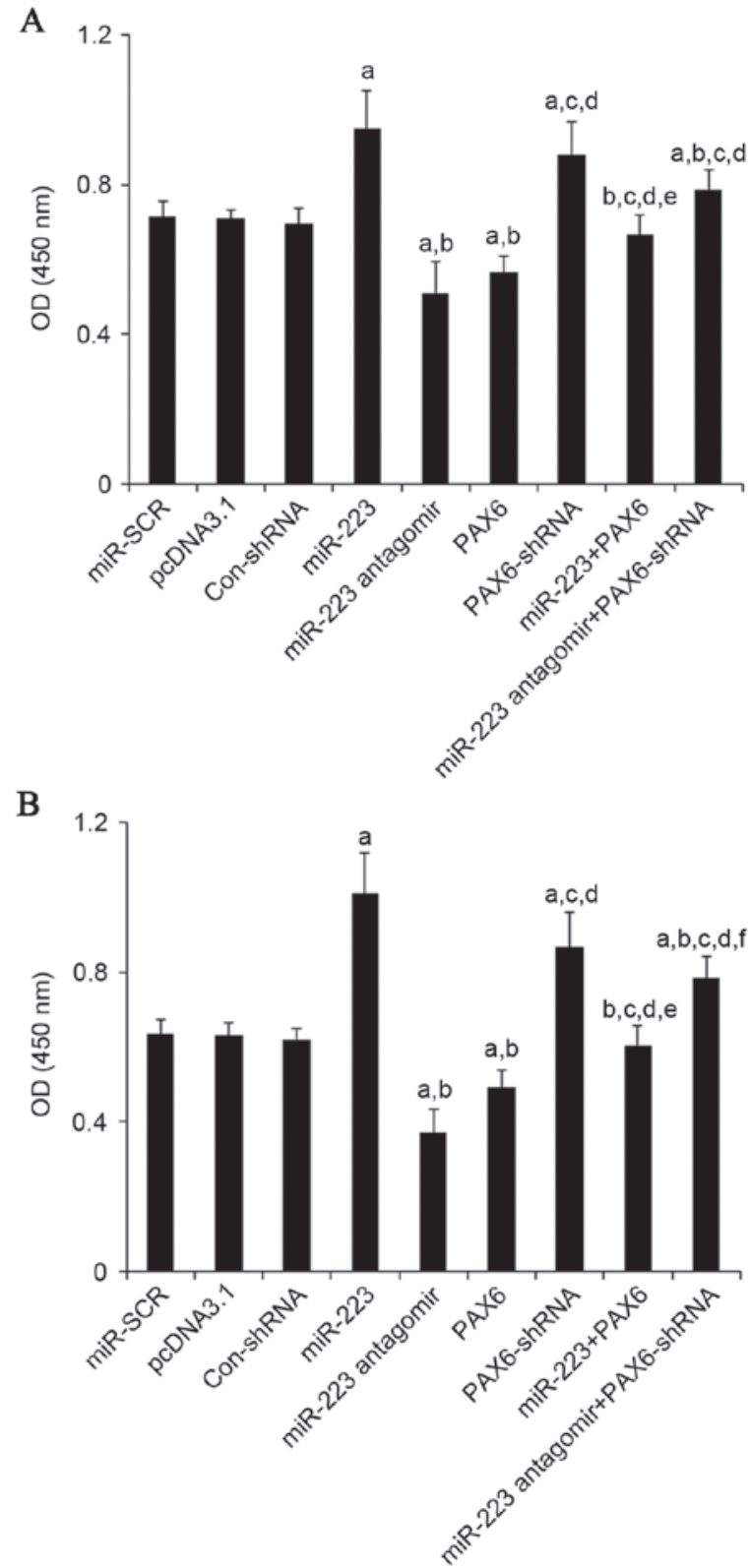

Figure 7. Effect of TMZ/miR-223/ PAX6 signaling on proliferation of GBM cells. In (A) U251 and (B) U118 GBM cells with TMZ treatment $(400 \mu \mathrm{M})$ for $48 \mathrm{~h}$, cell proliferation was measured at $48 \mathrm{~h}$ of culture with a microplate reader-based BrdU cell proliferation ELISA kit in cells transfected with SCR, cells transfected with the empty pcDNA3.1 plasmid, cells transfected with Con-shRNA, cells transfected with miR-223 mimic, cells transfected with miR-223 antagomir, cells transfected with PAX6, cells transfected with PAX6-shRNA, cells co-transfected with miR-223 mimic and PAX6 and cells co-transfected with miR-223 antagomir and PAX-shRNA. Cell proliferation was indicated by $\mathrm{OD}$ values at $450 \mathrm{~nm}$. ${ }^{\mathrm{a}} \mathrm{P}<0.05$ vs. controls (SCR, pcDNA3.1 and Con-shRNA); ${ }^{\circ} \mathrm{P}<0.05$ vs. miR-223; ${ }^{\circ} \mathrm{P}<0.05$ vs. miR-223 antagomir; ${ }^{\mathrm{d}} \mathrm{P}<0.05$ vs. $\mathrm{PAX6}$; ${ }^{\mathrm{P}} \mathrm{P}<0.05$ vs. PAX6-shRNA; ${ }^{\mathrm{f}} \mathrm{P}<0.05$ vs. miR-223 + PAX6. TMZ, temozolomide; miR, miRNA; PAX6, paired box 6; GBM, glioblastoma multiforme; SCR, scrambled control; Con, control; shRNA, short hairpin RNA; OD, optical density.

miR-223 inhibits non-small cell lung cancer cell proliferation and invasion, suggesting that it functions as a tumor suppressor (22). On the other hand, miR-223 is upregulated in bladder cancer (23) and esophageal squamous cell carcinoma (24), and it has been identified to promote tumor cell proliferation and invasion in gastric cancer and $\operatorname{GBM}(8,25)$. In this study, it is demonstrated that miR-223 antagonizes
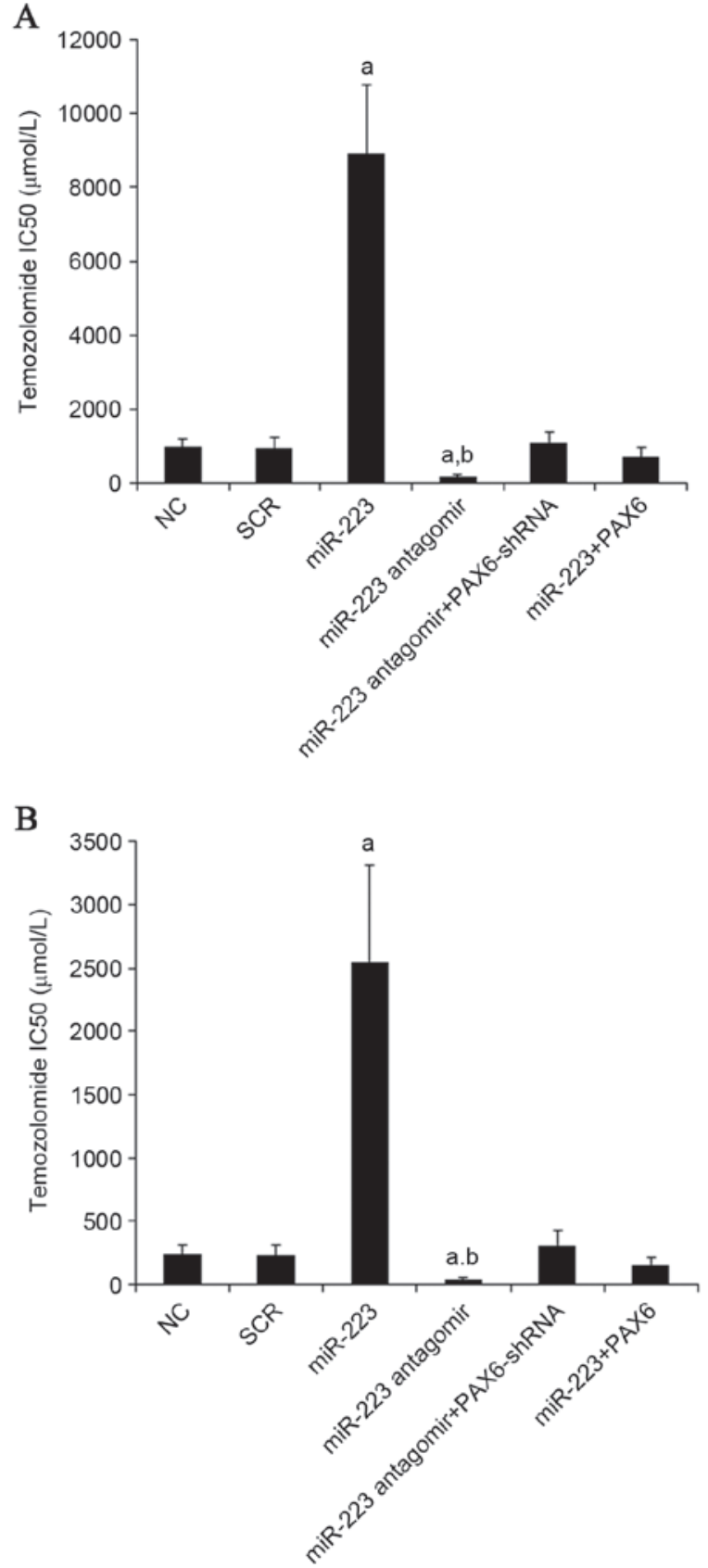

Figure 8. Effect of miR-223/PAX6 signaling on TMZ chemoresistance in GBM cells. (A) U251 and (B) U118 GBM cells were plated into 96-well plates. Transfection of plasmids, miR-223 mimic and antagomir were performed $6 \mathrm{~h}$ later. After $24 \mathrm{~h}$ of incubation, the medium was replaced by fresh medium with or without various concentrations of TMZ, and cell viability was assayed $72 \mathrm{~h}$ later. The half maximal inhibitory concentrations $\left(\mathrm{IC}_{50}\right)$ of TMZ were determined in NC cells, cells transfected with SCR, cells transfected with miR-223 mimic, cells transfected with miR-223 antagomir, cells co-transfected with miR-223 antagomir and PAX6-shRNA and cells co-transfected with miR-223 mimic and PAX6. ${ }^{\mathrm{a} P}<0.05$ vs. NC and SCR; ${ }^{\mathrm{b}} \mathrm{P}<0.05$ vs. miR-223. miR, miRNA; PAX6, paired box 6; TMZ, temozolomide; NC, normal control; SCR, scrambled control.

the inhibitory effects of TMZ on GBM cells by inhibiting the expression of PAX6. Taken together, the observations suggest that miR-223 likely serves a dual role in cancer malignancy and chemoresistance, depending on tissue specificity, and possibly, tissue-specific expression of PAX6. Due to the fact that PAX6 is expressed in the eye, brain and pancreas in healthy adults (5), the exploration of the role of 
miR-223/PAX6 signaling in the pathogenesis and chemoresistance of retinoblastoma and pancreatic cancer besides GBM may be beneficial.

Similar to that of several other solid tumor types, GBM is considered to be driven by a small sub-population of cells known as glioma stem cells (26). miR-223 reportedly functions as an essential regulator of human embryonic stem cell differentiation (27). Previous studies have indicated that PAX6 serves an important role in maintaining retinal stem cell properties (28) and is overexpressed in cancer stem-like cells in retinoblastoma (29), suggesting that PAX6 may be closely involved in maintenance of cancer stem cells. Thus, the examination of the role of miR-223/PAX6 signaling in the biology and chemoresistance of glioma stem cells may be beneficial in future studies.

In conclusion, the present study demonstrates that TMZ inhibits GBM cell proliferation by inhibiting the expression of miR-223, which leads to increased expression of tumor suppressor PAX6. While overexpression of miR-223 increases TMZ chemoresistance, inhibition of miR-223 with antagomir markedly decreases TMZ chemoresistance in GBM cells. The present study provides novel insight into the molecular mechanisms underlying the pharmacological effects and chemoresistance of TMZ for GBM.

\section{References}

1. Zhang Z and Lin CC: Taking advantage of neural development to treat glioblastoma. Eur J Neurosci 40: 2859-2866, 2014.

2. Wen PY and Kesari S: Malignant gliomas in adults. N Engl J Med 359: 492-507, 2008

3. van den Bent MJ, Hegi ME and Stupp R: Recent developments in the use of chemotherapy in brain tumours. Eur J Cancer 42 : $582-588,2006$

4. Giese A, Bjerkvig R, Berens ME and Westphal M: Cost of migration: Invasion of malignant gliomas and implications for treatment. J Clin Oncol 21: 1624-1636, 2003.

5. Zhou YH, Tan F, Hess KR and Yung WK: The expression of PAX6, PTEN, vascular endothelial growth factor, and epiderma growth factor receptor in gliomas: Relationship to tumor grade and survival. Clin Cancer Res 9: 3369-3375, 2003.

6. Zhou YH, Wu X, Tan F, Shi YX, Glass T, Liu TJ, Wathen K, Hess KR, Gumin J, Lang F and Yung WK: PAX6 suppresses growth of human glioblastoma cells. J Neurooncol 71: 223-229, 2005.

7. Mayes DA, Hu Y, Teng Y, Siegel E, Wu X, Panda K, Tan F, Yung WK and Zhou YH: PAX6 suppresses the invasiveness of glioblastoma cells and the expression of the matrix metalloproteinase-2 gene. Cancer Res 66: 9809-9817, 2006.

8. Huang BS, Luo QZ, Han Y, Li XB, Cao LJ and Wu LX: microRNA-223 promotes the growth and invasion of glioblastoma cells by targeting tumor suppressor PAX6. Oncol Rep 30: 2263-2269, 2013.

9. Ma R, Jiang T and Kang X: Circulating microRNAs in cancer: Origin, function and application. J Exp Clin Cancer Res 31: 38. 2012 .

10. Jones KB, Salah Z, Del Mare S, Galasso M, Gaudio E, Nuovo GJ, Lovat F, LeBlanc K, Palatini J, Randall RL, et al: miRNA signatures associate with pathogenesis and progression of osteosarcoma. Cancer Res 72: 1865-1877, 2012.

11. Hermansen SK and Kristensen BW: MicroRNA biomarkers in glioblastoma. J Neurooncol 114: 13-23, 2013.
12. Shi L, Chen J, Yang J, Pan T, Zhang S and Wang Z: MiR-21 protected human glioblastoma U87MG cells from chemotherapeutic drug temozolomide induced apoptosis by decreasing Bax/Bcl-2 ratio and caspase-3 activity. Brain Res 1352: 255-264, 2010.

13. Zhang S, Wan Y, Pan T, Gu X, Qian C, Sun G, Sun L, Xiang Y, Wang $\mathrm{Z}$ and Shi L: MicroRNA-21 inhibitor sensitizes human glioblastoma U251 stem cells to chemotherapeutic drug temozolomide. J Mol Neurosci 47: 346-356, 2012.

14. Wang J, Sai K, Chen FR and Chen ZP: miR-181b modulates glioma cell sensitivity to temozolomide by targeting MEK1. Cancer Chemother Pharmacol 72: 147-158, 2013.

15. Mesti T, Savarin P, Triba MN, Le Moyec L, Ocvirk J, Banissi C and Carpentier AF: Metabolic impact of anti-angiogenic agents on U87 glioma cells. PLoS One 9: e99198, 2014.

16. Olivares S, Green RM and Henkel AS: Endoplasmic reticulum stress activates the hepatic activator protein 1 complex via mitogen activated protein kinase-dependent signaling pathways. PLoS One 9: e103828, 2014.

17. Ding X, Zhang Z, Li S and Wang A: Combretastatin A4 phosphate induces programmed cell death in vascular endothelial cells. Oncol Res 19: 303-309, 2011.

18. Louis DN, Ohgaki H, Wiestler OD, Cavenee WK, Burger PC, Jouvet A, Scheithauer BW and Kleihues P: The 2007 WHO classification of tumours of the central nervous system. Acta Neuropathol 114: 97-109, 2007.

19. Fazi F, Rosa A, Fatica A, Gelmetti V, De Marchis ML, Nervi C and Bozzoni I: A minicircuitry comprised of microRNA-223 and transcription factors NFI-A and C/EBPalpha regulates human granulopoiesis. Cell 123: 819-831, 2005

20. Stamatopoulos B, Meuleman N, Haibe-Kains B, Saussoy P, Van Den Neste E, Michaux L, Heimann P, Martiat P, Bron D and Lagneaux L: microRNA-29c and microRNA-223 down-regulation has in vivo significance in chronic lymphocytic leukemia and improves disease risk stratification. Blood 113: 5237-5245, 2009.

21. Wong QW, Lung RW, Law PT, Lai PB, Chan KY, To KF and Wong N: MicroRNA-223 is commonly repressed in hepatocellular carcinoma and potentiates expression of Stathmin1. Gastroenterology 135: 257-269, 2008.

22. Nian W, Ao X, Wu Y, Huang Y, Shao J, Chen Z, Chen F and Wang D: miR-223 functions as a potent tumor suppressor of the Lewis lung carcinoma cell line by targeting insulin-like growth factor-1 receptor and cyclin-dependent kinase 2. Oncol Lett 6: 359-366, 2013.

23. Gottardo F, Liu CG, Ferracin M, Calin GA, Fassan M, Bassi P, Sevignani C, Byrne D, Negrini M, Pagano F, et al: Micro-RNA profiling in kidney and bladder cancers. Urol Oncol 25: 387-392, 2007.

24. Kurashige J, Watanabe M, Iwatsuki M, Kinoshita K, Saito S, Hiyoshi Y, Kamohara H, Baba Y, Mimori K and Baba H: Overexpression of microRNA-223 regulates the ubiquitin ligase FBXW7 in oesophageal squamous cell carcinoma. Br J Cancer 106: 182-188, 2012.

25. Li X, Zhang Y, Zhang H, Liu X, Gong T, Li M, Sun L, Ji G, Shi Y, Han Z, et al: miRNA-223 promotes gastric cancer invasion and metastasis by targeting tumor suppressor EPB41L3. Mol Cancer Res 9: 824-833, 2011.

26. Singh SK, Hawkins C, Clarke ID, Squire JA, Bayani J, Hide T, Henkelman RM, Cusimano MD and Dirks PB: Identification of human brain tumour initiating cells. Nature 432: 396-401, 2004.

27. Yu YH, Zhang L, Wu DS, Zhang Z, Huang FF, Zhang J, Chen XP, Liang DS, Zeng $\mathrm{H}$ and Chen FP: MiR-223 regulates human embryonic stem cell differentiation by targeting the IGF-1R/Akt signaling pathway. PLoS One 8: e78769, 2013.

28. Bhattacharya S, Das A, Mallya K and Ahmad I: Maintenance of retinal stem cells by Abcg2 is regulated by notch signaling. J Cell Sci 120: 2652-2662, 2007.

29. Ma B, Lei X, Guan Y, Mou LS, Yuan YF, Yue H, Lu Y, Xu GT and Qian J: Maintenance of retinal cancer stem cell-like properties through long-term serum-free culture from human retinoblastoma. Oncol Rep 26: 135-143, 2011. 